\title{
Effective-mass Klein-Gordon Equation for non-PT/non-Hermitian Generalized Morse Potential
}

\author{
Altug $\operatorname{Arda}^{1, * *}$ and Ramazan Sever $^{2,+\dagger}$ \\ ${ }^{1}$ Department of Physics Education, Hacettepe University, 06800, Ankara,Turkey \\ ${ }^{2}$ Department of Physics, Middle East Technical University, 06531, Ankara,Turkey
}

(Dated: October 25, 2018)

\begin{abstract}
The one-dimensional effective-mass Klein-Gordon equation for the real, and non-PTsymmetric/non-Hermitian generalized Morse potential is solved by taking a series expansion for the wave function. The energy eigenvalues, and the corresponding eigenfunctions are obtained. They are also calculated for the constant mass case.

Keywords: position dependent mass, Klein-Gordon Equation, generalized Morse potential, PTsymmetry, energy eigenvalues, eigenfunctions.

PACS numbers: 03.65.Fd, 03.65.Ge
\end{abstract}

*E-mail: arda@hacettepe.edu.tr

†E-mail: sever@metu.edu.tr 


\section{INTRODUCTION}

In the past few decades, there has been an increased interest to find the exact solutions of the non-relativistic and relativistic equations. The solutions of the Schrödinger equation has been studied by using different methods based on perturbative and non-perturbative approaches [1-5]. The Klein-Gordon (KG) and Dirac equations have been also studied for different type of potentials such as Aharonov-Bohm (AB) potential [6], the AB plus Dirac monopole potential [7, 11], generalized Hulthen, harmonic, and linear potentials, generalized asymmetrical Hartmann potentials, for a uniform magnetic field, pseudoharmonic oscillator, and exponential-type potentials [12-20].

Recently, there has been considerable work on the PT-symmetric quantum mechanics. Following the initially studies of Bender et al. [21], the PT-symmetric theory has been successfully studied by many authors because of their importance, and also for using in different research areas, such as quantum field theory, and nuclear physics [22, 23]. In the present study, we take into account the form of the generalized Morse potential having also non-PT symmetric property to achieve the solutions within the position-dependent mass formalism, and study the effects of the mass varying with spatially coordinate on the solutions of the case of constant mass. It could be interested to solve the relativistic KG equation in this point of view.

The solutions of the above wave equations are taken in general for constant or timedependent masses $[24,25]$. There are also many examples of physically important systems, for which the mass depends on coordinates. So far, solving the above equations for the case of spatially dependent mass has been studied by many authors [26-34] for different types of the mass distributions such as an exponentially, and quadratic mass functions [27], inverse-quadratic dependence of mass [35], trigonometric mass-distributions [36], and mass functions of the forms $\mathrm{m}(\mathrm{r})=\mathrm{r}^{\alpha}$, especially for three-dimensional problems [36, 37]. We will use also a mass distribution having an exponential form, and study in the half-plane to achieve a physically acceptable (finite) mass function, which has a decreasingly behavior in this range.

In the present work, we study the bound state solutions of the KG equation with real and non-PT symmetric/non-Hermitian forms of generalized Morse potential in the case of a coordinate-dependence mass distribution. In order to find the energy spectra, and the 
corresponding wave functions we prefer to use an infinite series for the wave function. This approach is a powerful technique to solve second order differential equations especially for the potential forms having two and/or more terms [38, 39]. We obtain also the energy spectrum, and the corresponding eigenfunctions in the case of constant mass for two potential types.

The organization of the work as follows. In section II, we obtain the exact bound-state energy spectra for real, and non-PT symmetric/non-Hermitian cases of generalized Morse potential, and the corresponding eigenfunctions by taking an infinite series for the wave function in the case of a convenient mass distribution. We summarize the numerical results in one table, and two figures to see the effects of the mass depending on coordinate. For this aim we do the numerical analysis for three different diatomic molecules such as $\mathrm{H}_{2}$, $\mathrm{LiH}$, and $\mathrm{HCl}$. We give also the results for the case of constant mass, and present our conclusions in Section III.

\section{BOUND STATE SOLUTIONS}

\section{A. Real Generalized Morse Case}

The one-dimensional KG equation is written in terms of scalar and vector potential as following [40]

$$
\frac{\mathrm{d}^{2} \psi(x)}{\mathrm{d} x^{2}}+\frac{1}{\hbar^{2} c^{2}}\left[\left[E-V_{v}(x)\right]^{2}-\left[m c^{2}+V_{s}(x)\right]^{2}\right] \psi(x)=0,
$$

where $\mathrm{m}$ is the mass of the particle, $\mathrm{E}$ denotes the energy, and $\mathrm{V}_{s}(x)$ and $\mathrm{V}_{v}(x)$ are the scalar and vector parts of the potential, respectively, and $c$ is the velocity of light. In the absence of the vector potential, the KG equation can be written as

$$
\frac{\mathrm{d}^{2} \psi(x)}{\mathrm{d} x^{2}}+\frac{2 m}{\hbar^{2}}\left(\varepsilon-\frac{V_{s}^{2}(x)}{2 m c^{2}}-V_{s}(x)\right) \psi(x)=0,
$$

which is a Schrödinger-like equation with the energy eigenvalue $\varepsilon=\frac{E^{2}}{2 m c^{2}}-\frac{m c^{2}}{2}$.

We consider the scalar potential $\mathrm{V}_{s}(x)$ in Eq. (2) as generalized Morse potential, which can be used to describe the vibrations of a two-atomic molecule, as the following

$$
V_{s}(x)=V_{1} \mathrm{e}^{-2 \beta x}-V_{2} \mathrm{e}^{-\beta x},(0 \leq x \leq+\infty)
$$


where $\mathrm{V}_{1}$, and $\mathrm{V}_{2}$ are two real parameters, and $x=\left(r-r_{0}\right) / r_{0}$. The parameter $\beta$ is $\operatorname{ar}_{0}$, here, $\mathrm{a}$ is the potential width, and $\mathrm{r}_{0}$ is the equilibrium distance.

Here, we prefer to use the following mass-distribution

$$
m(x)=m_{0}+m_{1} \mathrm{e}^{-\beta x},
$$

where $\mathrm{m}_{0}$, and $\mathrm{m}_{1}$ are real parameters. This distribution is finite at infinity, and enables us to analyze the results in the case of constant mass.

Substituting Eqs. (3) and (4) into Eq. (2), than we have

$$
\begin{aligned}
\frac{\mathrm{d}^{2} \psi(x)}{\mathrm{d} x^{2}} & +\left\{\frac{1}{\hbar^{2} c^{2}}\left[E^{2}-m_{0}^{2} c^{4}\right]+\frac{2}{\hbar^{2} c^{2}}\left[V_{2} m_{0} c^{2}-m_{0} m_{1} c^{4}\right] \mathrm{e}^{-\beta x}\right. \\
& -\frac{1}{\hbar^{2} c^{2}}\left[V_{2}^{2}+2 V_{1} m_{0} c^{2}-2 V_{2} m_{1} c^{2}+m_{1}^{2} c^{4}\right] \mathrm{e}^{-2 \beta x} \\
& \left.+\frac{2}{\hbar^{2} c^{2}}\left[V_{1} V_{2}-V_{1} m_{1} c^{2}\right] \mathrm{e}^{-3 \beta x}-\frac{V_{1}^{2}}{\hbar^{2} c^{2}} \mathrm{e}^{-4 \beta x}\right\} \psi(x)=0,
\end{aligned}
$$

By using the transformations on coordinate and wave function

$$
z=\mathrm{e}^{-\beta x}, \quad \psi(z)=\frac{1}{\sqrt{z}} \phi(z)
$$

and with the help of the following parameters

$$
\begin{aligned}
& A_{1}=Q^{2}\left(E^{2}-m_{0}^{2} c^{4}\right)+\frac{1}{4}, \\
& A_{2}=2 Q^{2}\left(V_{2} m_{0} c^{2}-m_{0} m_{1} c^{4}\right), \\
& A_{3}=Q^{2}\left(V_{2}^{2}+2 V_{1} m_{0} c^{2}-2 V_{2} m_{1} c^{2}+m_{1}^{2} c^{4}\right), \\
& A_{4}=2 Q^{2}\left(V_{1} V_{2}-V_{1} m_{1} c^{2}\right), \\
& A_{5}=Q^{2} V_{1}^{2} .
\end{aligned}
$$

we get

$$
\frac{\mathrm{d}^{2} \phi(z)}{d z^{2}}+\left(-A_{3}+\frac{A_{1}}{z^{2}}+\frac{A_{2}}{z}-A_{4} z-A_{5} z^{2}\right) \phi(z)=0 .
$$


where $\mathrm{Q}^{2}=1 / \hbar^{2} c^{2} \beta^{2}$ in the above expressions $(0 \leq z \leq 1)$. It would be interested the results obtained from the last equation for a special case $\mathrm{A}_{2}=\mathrm{A}_{4}=0$, which gives

$$
\frac{\mathrm{d}^{2} \phi(z)}{d z^{2}}+\left(\frac{A_{1}}{z^{2}}-A_{5} z^{2}\right) \phi(z)=A_{3} \phi(z)
$$

This equation has a similar form with the ones given by Eq. (7) in Ref. [17] for $\mathrm{A}_{5} \rightarrow \alpha^{2}$, $\mathrm{A}_{1} \rightarrow-\ell^{\prime}\left(\ell^{\prime}+1\right)$, and $\mathrm{A}_{3} \rightarrow-\lambda\left(\alpha, \ell^{\prime}\right.$, and $\lambda$ are the parameters used in Ref. [17]). Eq. (9) corresponds to the equation of the harmonic oscillator with centrifugal potential barrier, so we could give the solutions as

$$
\varepsilon_{n}=\mp\left\{m_{0}^{2} c^{4}+\frac{4 c^{2}}{\beta^{2} \hbar^{2}}\left(m_{1} c^{2}+m_{0}\right)^{2}\left[2 n+1+\sqrt{\frac{1}{4}-V_{1}^{2} Q^{2}}\right]^{-2}-\frac{1}{4 Q^{2}}\right\}^{1 / 2} .
$$

We turn to the solution of Eq. (8), and write the wave function as $[38,39]$

$$
\phi(z)=\mathrm{e}^{p z+(1 / 2) q z^{2}} \sum_{n=0}^{\infty} a_{n} z^{2 n+L+1 / 2} .
$$

Substituting Eq. (11) into Eq. (8), and equating of the coefficients to zero, we get the following identities among coefficients, and the expressions for $\mathrm{p}$, and $\mathrm{q}$

$$
X_{n} a_{n}+Y_{n+1} a_{n+1}+Z_{n+2} a_{n+2}=0,
$$

where

$$
\begin{aligned}
X_{n} & =2 q(2 n+L+1)-A_{3}, \\
Y_{n} & =A_{2}+p(4 n+2 L+1), \\
Z_{n} & =4 n(n+L)+2 L^{2} .
\end{aligned}
$$

and

$$
\begin{gathered}
q^{2}=A_{5} \\
2 p q=A_{4} .
\end{gathered}
$$


where the new parameter $\mathrm{L}$ is defined as $\mathrm{L}^{2} \rightarrow Q^{2}\left(E^{2}-m_{0}^{2} c^{4}\right)$, and we choose the parameters as $\mathrm{p}=Q\left(V_{2}-m_{1} c^{2}\right)$, and $\mathrm{q}=-Q V_{1}$ to obtain a physically solution.

On the other hand, $\mathrm{X}_{n}, \mathrm{Y}_{n}$ and $\mathrm{Z}_{n}$ must satisfy the following condition to determine the coefficients in the system of equations given in Eqs. (13)-(15)

$$
\operatorname{det}\left|\begin{array}{cccccc}
Y_{0} & Z_{1} & \ldots & \ldots & \ldots & 0 \\
X_{0} & Y_{1} & Z_{2} & \ldots & \ldots & 0 \\
X_{1} & Y_{2} & Z_{3} & \ldots & \ldots & 0 \\
\vdots & \vdots & \vdots & \ddots & \vdots & \vdots \\
0 & 0 & 0 & 0 & X_{n-1} & Y_{n}
\end{array}\right|=0
$$

In the case of $\mathrm{a}_{n} \neq 0$, but $\mathrm{a}_{n+1}=\mathrm{a}_{n+2}=\ldots=0$ in Eq. (12), we impose $\mathrm{X}_{n}=0$. This leads to the following algebraic equation

$$
2 q(2 n+L+1)=A_{3} .
$$

Substituting the values of the parameters defined in Eq. (7) into Eq. (19), and by using p, and q obtained from Eqs. (16)-(17), we have the energy eigenvalues of the generalized Morse potential as

$$
E_{n}= \pm\left\{m_{0}^{2} c^{4}+\frac{1}{Q^{2}}\left[2 n+1+Q \tilde{V}_{1}\right]^{2}+\frac{m_{1} c^{2} \tilde{V}_{2}}{Q}\left[2(2 n+1)+2 Q \tilde{V}_{1}+Q m_{1} c^{2} \tilde{V}_{2}\right]\right\}^{1 / 2}
$$

where

$$
\tilde{V}_{1}=\frac{V_{2}^{2}}{2 V_{1}}+m_{0} c^{2}, \quad \tilde{V}_{2}=-\frac{V_{2}}{V_{1}}+\frac{m_{1} c^{2}}{2 V_{1}}
$$

It is seen that the energy levels of the particle and antiparticles are split around zero. On the other hand, the energy spectrum of the generalized Morse potential for the positiondependent mass is real. The part of the energy eigenvalues coming from the coordinate dependence of the mass is dependent on the quantum number $\mathrm{n}$, and also on the potential parameters $\mathrm{V}_{1}$, and $\mathrm{V}_{2}$. 
The corresponding wave functions can be written as the following

$$
\phi_{n}(z)=\left(a_{0} z^{1 / 2}+a_{1} z^{5 / 2}+\ldots\right) z^{L} \exp \left[Q\left(V_{2}-m_{1} c^{2}\right) z-\frac{Q V_{1}}{2} z^{2}\right]
$$

where $a_{i}(i=0,1 \ldots)$ are the coefficients of the series.

In order to obtain the energy spectrum of the real generalized Morse potential for the case of constant mass, we have to set $\mathrm{m}_{1}=0$ in Eq. (20), than we get

$$
E_{n}^{m_{1}=0}= \pm\left(2 n+1+Q \tilde{V}_{1}\right) \sqrt{\frac{1}{Q^{2}}+\left(\frac{m_{0} c^{2}}{2 n+1+Q \tilde{V}_{1}}\right)^{2}}
$$

According to Eq. (23), the energy levels of the particle and antiparticles are symmetric about the zero in the limit of the constant mass. On the other hand, the energy spectrum of the real generalized Morse potential is purely real as in the first case.

We summarize the numerical results in Table I for the $\mathrm{H}_{2}, \mathrm{LiH}$, and $\mathrm{HCl}$ molecules in the case of the constant mass $\mathrm{m}_{0}$ obtained from Eq. (23). We give the bound state energies of the particles $\left(+\mathrm{E}_{n}\right)$, and antiparticles $\left(-\mathrm{E}_{n}\right)$ for different n-values in Table I. Further, we plot the dependence of the ground states of the above molecules on $\mathrm{m}_{1}$ for $\mathrm{H}_{2}$, and $\mathrm{LiH}$, $\mathrm{HCl}$ molecules in Fig.1, and Fig.2, respectively. We do the numerical analysis in the range

$10^{-6}<\frac{1}{M}<10^{-4}$, where $\mathrm{M}=\mathrm{m}_{0} / \mathrm{m}_{1}$, and $(\mathrm{p})$ denotes the particle case, and (a) denotes the antiparticle one in figures.

The corresponding eigenfunctions in the case of the constant mass can be obtained from Eq. (22) by setting $\mathrm{m}_{1}=0$.

\section{B. Non-PT Symmetric and non-Hermitian Generalized Morse Case}

Now we consider the complex case of the generalized Morse potential, where the potential parameters are written as

$$
\begin{aligned}
& V_{1}=v_{1}^{2}-v_{2}^{2}+2 \mathrm{i} v_{1} v_{2} \\
& V_{2}=v_{1}+\mathrm{i} v_{2}+2 v_{3}\left(v_{1}+\mathrm{i} v_{2}\right)
\end{aligned}
$$


where $v_{\mathrm{i}}^{\prime} s(\mathrm{i}=1,2,3)$ are the real parameters, and $\beta=1$. This complex form of the generalized Morse potential is called non-PT symmetric/non-Hermitian potential form of the potential $[41,42]$.

Substituting Eq. (24) into Eq. (21), than we have from Eq. (20) for the energy spectra of the non-PT symmetric/non-Hermitian generalized Morse potential the following

$E_{n}= \pm\left\{m_{0}^{2} c^{4}+\frac{1}{Q^{\prime 2}}\left[2 n+1+Q^{\prime} \tilde{V}_{1}^{\prime}\right]^{2}+\frac{m_{1} c^{2} \tilde{V}_{2}^{\prime}}{Q^{\prime}}\left[2(2 n+1)+2 Q^{\prime} \tilde{V}_{1}^{\prime}+Q^{\prime} m_{1} c^{2} \tilde{V}_{2}^{\prime}\right]\right\}^{1 / 2}$

where

$$
\tilde{V}_{1}^{\prime}=\frac{2\left(v_{3}+1\right)^{2}}{2}+m_{0} c^{2}, \quad \tilde{V}_{2}^{\prime}=\frac{2 v_{3}+1}{v_{1}+\mathrm{i} v_{2}}+\frac{m_{1} c^{2}}{2\left(v_{1}+\mathrm{i} v_{2}\right)^{2}}, \quad Q^{\prime}=\frac{1}{\hbar^{2} c^{2}} .
$$

The corresponding eigenfunctions can be written as

$$
\phi_{n}(z)=\left(a_{0} z^{L+1 / 2}+a_{1} z^{L+5 / 2}+\ldots\right) \exp \left[Q\left(V_{2}-m_{1} c^{2}\right) z-\frac{Q V_{1}}{2} z^{2}\right]
$$

The energy spectrum for the case of constant mass is given as

$$
E_{n}^{m_{1}=0}= \pm\left(2 n+1+Q^{\prime} \tilde{V}_{1}^{\prime}\right) \sqrt{\frac{1}{Q^{\prime 2}}+\left(\frac{m_{0} c^{2}}{2 n+1+Q^{\prime} \tilde{V}_{1}}\right)^{2}}
$$

It is clearly seen that the energy eigenvalues of the non-PT symmetric/non-Hermitian generalized Morse potential has real and imaginary parts, which is coming from the coordinate dependence of the mass, but in the constant mass limit the energy spectrum is real. On the other hand, the energy levels of the non-PT symmetric/non-Hermitian generalized Morse potential is symmetric about zero as in the real potential case. The eigenfunctions for the case of constant mass are obtained by setting $\mathrm{m}_{1}=0$ in Eq. (27).

\section{CONCLUSION}

We have obtained the solutions of the $\mathrm{KG}$ equation for the real and non-PT symmetric/non-Hermitian generalized Morse potential with position dependent mass. The 
energy spectra and the corresponding wave functions have been obtained by using an infinite series for the wave function. We have found that the real generalized Morse potential has a real energy eigenvalues in the case of the position-dependent mass. We have summarized first the energy eigenvalues of three diatomic molecules for different values of $n$, and than plot two different graphs where the energy eigenvalues varying with mass to see the effects of the mass depending on spatially coordinate. We have also studied the non-PT symmetric/non-Hermitian case of the potential, and pointed out that the energy eigenvalues are imaginary in this case. We have found that the energy levels are purely real in the case of the constant mass as given in Eqs. (23), and (28) for both form of the generalized Morse potential.

\section{ACKNOWLEDGMENTS}

This research was partially supported by the Scientific and Technical Research Council of Turkey. 
[1] J. P. Killingbeck, A. Grosjean, and G. Jolicard, J. Phys. A: Math. Gen. 34, 8309 (2001).

[2] A. Arda, Turk. J. Phys. 28, 223 (2004).

[3] J. Chen, L. C. Kweck, and C. H. Oh, Phys. Rev. A 67, 012101 (2003).

[4] S. N. Biswas et al., J. Math. Phys. 14, 1190 (1973).

[5] A. Khare, and U. P. Sukhatme, J. Phys. A 26, L901-L904 (1993) [arXiv: hep-th/9212147].

[6] C. R. Hagen, Phys. Rev. D 48, 5935 (1993), [arXiv: hep-th/9308009].

[7] V. M. Villalba, [arXiv: hep-th/9409102].

[8] A. S.de Castro, Int. J. Mod. Phys. A 22, 2609 (2007) [arXiv: hep-th/0511010].

[9] A. S.de Castro, Phys. Lett. A 338, 81 (2005), [arXiv: hep-th/0502201].

[10] A. S.de Castro, Phys. Lett. A 346, 71 (2005), [arXiv: hep-th/0507218].

[11] A. S.de Castro, Phys. Lett. A 342, 53 (2005) [arXiv: hep-th/0409216].

[12] W. C. Qiang, R.-S. Zhou, and Y. Gao, Phys. Lett. A 371, 201 (2007).

[13] N. Saad, [arXiv: math-ph/0709.4014].

[14] R. Giachetti, and E. Sorace, Phys. Rev. Lett. 101, 190401 (2008) [arXiv: hep-th/0706.0127].

[15] K. Bhattacharya, [arXiv: hep-th/0705.4275].

[16] A. S. Dutra, and M. B. Hott, [arXiv: quant-ph/0705.3447].

[17] C. Gang, C. Zi-Dong, and L. Zhi-Mei, Chi. Phys. 13, 279 (2004).

[18] C. Gang, Phys. Lett. A 339, 300 (2005).

[19] C. Gang, Acta. Phys. Sin. 50, 1651 (2001).

[20] L. Z. Yi, Y. F. Diao, J. Y. Liu, and C. S. Jia, Phys. Lett. A 333, 212 (2004).

[21] C. M . Bender, S. Boettcher, and P. N. Meisinger, J. Math. Phys. 40, 2201 (1999).

[22] S. M. Ikhdair, and R. Sever, Ann. Phys. 16, 218 (2007).

[23] C. S. Jia, P. Q. Wang, J. Y. Liu, and S. He, Int. J. Theor. Phys. 47, 2513 (2008), and references therein.

[24] A. Mostafazadeh, J. Phys. A 31, 6495 (1998).

[25] A. Mostafazadeh, Phys. Rev. A 55, 4084 (1997).

[26] A. D. Alhaidari, Phys. Lett. A 322, 72 (2004).

[27] A.S. Dutra, and C. A. S. Almeida, Phys. Lett. A 275, 25 (2000).

[28] B. Gonul, B. Gonul, D. Tutcu, and O. Ozer, Mod. Phys. Lett. A 17, 2057 (2002). 
[29] B. Gonul, O. Ozer, B. Gonul, and F. Uzgun, Mod. Phys. Lett. A 17, 2453 (2002).

[30] B. Gonul, and M. Kocak, Chin. Phys. Lett. 20, 2742 (2005).

[31] B. Gonul, and M. Kocak, [arXiv: quant-ph/0512035].

[32] C. Tezcan, and R. Sever, J. Math. Chem. 42, 387 (2007) [arXiv: quant-ph/0604041].

[33] I. O. Vakarchuk, J. Phys. A: Math. Gen. 38, 4727 (2005).

[34] C. Gang, and Z. Chen, Phys. Lett. A 331, 312 (2004).

[35] L. Jiang, L.-Z. Yi, and C.-S. Jua, Phys. Lett. A 345, 249 (2005).

[36] A. D. Alhaidari, Phys. Rev. A 66, 042116 (2002).

[37] G. X. Ju, Y. Xiang, and Z. Z. Ren, [arXiv: quant-ph/0601005].

[38] S. H. Dong, Z. Ma, and G. Espozito, Found. Phys. Lett. 12, 465 (1999).

[39] S. H. Dong, Int. J. Theor. Phys. 39, 1119 (2000); 40, 569 (2001).

[40] A. S. de Castro, Phys. Lett. A 338, 81 (2005).

[41] Z. Ahmed, Phys. Lett. A 282, 343 (2001).

[42] G. Bagchi, and C. Quesne, Phys. Lett. A 273, 285 (2000).

[43] E. D. Filho, and R. M. Ricotta, Phys. Lett. A 269, 269 (2000) [arXiv: hep-th/9910254]. 
TABLE I: The dependence of bound states on $\mathrm{n}$ in $M e V$ for $\mathrm{H}_{2}\left(\mathrm{D}=38267.76 \mathrm{~cm}^{-1}, \mathrm{a}=1,9426 \AA^{-1}\right.$, $\mathrm{r}_{0}=0,7416 \AA$, and $\left.\mathrm{m}_{0}=0,50391 \mathrm{amu}\right), \operatorname{LiH}\left(\mathrm{D}=20287 \mathrm{~cm}^{-1}, \mathrm{a}=1,1280 \AA^{-1}, \mathrm{r}_{0}=1,5956 \AA\right.$, and $\left.\mathrm{m}_{0}=0,8801221 \mathrm{amu}\right)$, and $\mathrm{HCl}$ molecule $\left(\mathrm{D}=37255 \mathrm{~cm}^{-1}, \mathrm{a}=1,8677 \AA^{-1}, \mathrm{r}_{0}=1,2746 \AA\right.$, and $\left.\mathrm{m}_{0}=0,9801045 \mathrm{amu}\right)[43]$ with $\ell=0$.

\begin{tabular}{cccc}
\hline \hline$n$ & $\pm \mathrm{E}_{n}\left(\mathrm{H}_{2}\right)$ & $\pm \mathrm{E}_{n}(\mathrm{LiH})$ & $\pm \mathrm{E}_{n}(\mathrm{HCl})$ \\
0 & 663,819 & 1159,420 & 1291,130 \\
2 & 663,827 & 1159,430 & 1291,140 \\
4 & 663,835 & 1159,440 & 1291,150 \\
10 & 663,859 & 1159,470 & 1291,190 \\
20 & 663,899 & 1159,520 & 1291,260 \\
30 & 663,939 & 1159,570 & 1291,330 \\
40 & 663,979 & 1159,620 & 1291,390 \\
50 & 664,020 & 1159,670 & 1291,460 \\
\hline \hline
\end{tabular}




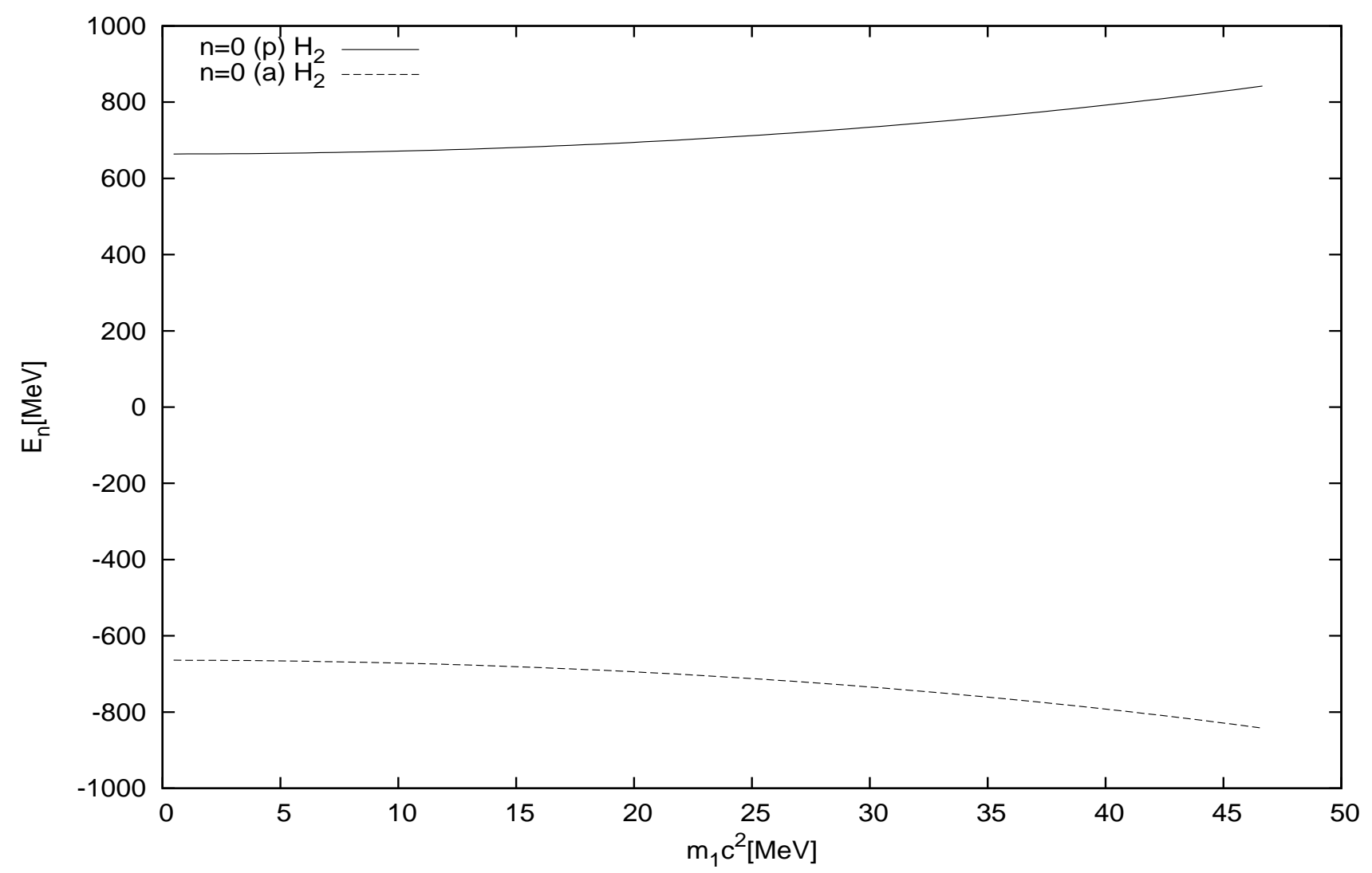

FIG. 1: The variation of ground states of the $\mathrm{H}_{2}$ molecule with $\mathrm{m}_{1}$. 


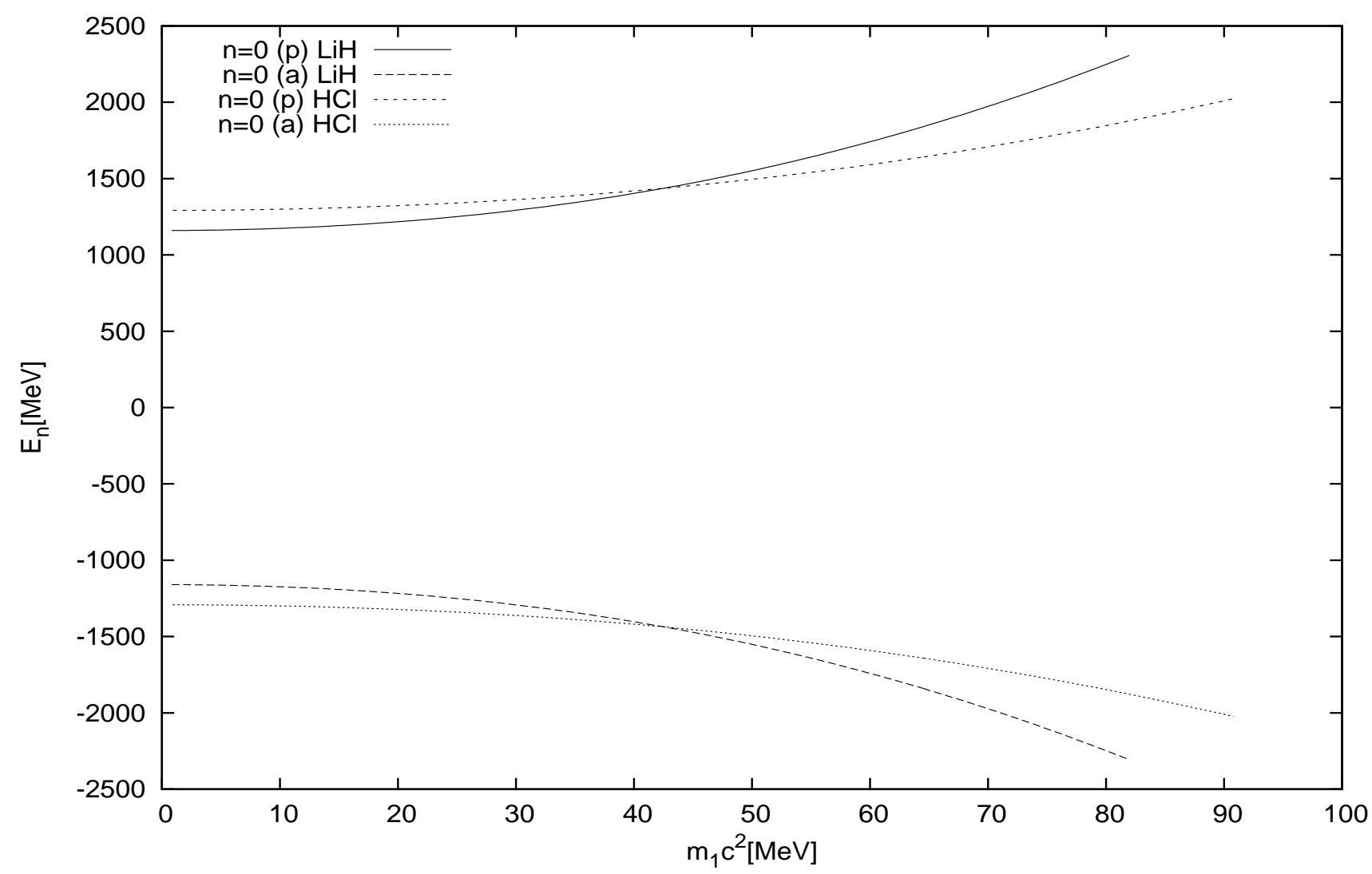

FIG. 2: The variation of ground states of the $\mathrm{LiH}$, and $\mathrm{HCl}$ molecules with $\mathrm{m}_{1}$. 